\title{
Surface-localized plasmon resonance in a system of randomly arranged gold nanorods on a dielectric substrate
}

\author{
${ }^{1 *}$ Yaremchuk I., ${ }^{2}$ Pidluzhna A., ${ }^{3}$ Stakhira P., ${ }^{4}$ Kuntyi O., ${ }^{4}$ Sus L., ${ }^{5}$ Savaryn V., \\ ${ }^{5}$ Kostruba A., ${ }^{1}$ Fitio V. and ${ }^{1,6}$ Bobitski Y. \\ ${ }^{1}$ Department of Photonics, Lviv Polytechnic National University, \\ S. Bandera Street 12, 79013 Lviv, Ukraine, iryna.y.yaremchuk@lpnu.ua \\ ${ }^{2}$ Department of Applied Physics and Nanomaterials Science, Lviv Polytechnic \\ National University, S. Bandera Street 12, 79013 Lviv, Ukraine \\ ${ }^{3}$ Department of Electronic Devices, Lviv Polytechnic National University, \\ S. Bandera Street 12, 79013 Lviv, Ukraine \\ ${ }^{4}$ Department of Chemistry and Chemical Technology, Lviv Polytechnic National \\ University, S. Bandera Street 12, 79013 Lviv, Ukraine \\ ${ }^{5}$ Department of Physics and Mathematics, Stepan Gzhytskyi National University of \\ Veterinary Medicine and Biotechnologies, 79010 Lviv, Ukraine \\ ${ }^{6}$ Department of Physics, College of Natural Sciences, Institute of Physics, \\ University of Rzeszow, Pigonia Street 1, 35959 Rzeszow, Poland
}

Received: 23.12 .2020

\begin{abstract}
Gold-nanorod arrays of a quasi-hexagonal shape are successfully obtained with electrochemical deposition method and their optical properties are investigated. The optical response of a two-layer structure, in which the first layer is a nanocomposite consisting of $\mathrm{Au}$ nanorods and the second one is a thin indium tin oxide film on a glass substrate, has been analyzed. Dependences of absorption crosssection of $\mathrm{Au}$ nanorods on the light wavelength at different eccentricities are modelled using electrostatic approximation. It is shown that longitudinal plasmon resonance prevails over other resonances for the Au nanorods deposited on glass substrates. A Maxwell-Garnett theory and a matrix method are used to predict the optical characteristics of the whole structure. We have demonstrated that it is possible to estimate the concentration of nanorods on the surface using the appropriate simulation results. In addition, efficient absorption properties can be obtained at a given wavelength by changing the geometry of nanorods. In particular, there is a shift of the absorption peak towards near-infrared region whenever the nanorods become high enough and smaller in diameter.
\end{abstract}

Keywords: plasmon resonance, nanoparticles, arrays of Au nanorods, dielectric permittivity

UDC: 517.958:535.14, 53.072:53:004

\section{Introduction}

Recently, a lot of scientific interest has been addressed to metallic nanostructures under resonance conditions, including a so-called localized surface plasmon resonance [1-3]. Oscillations of the conduction-band electrons determine the optical properties of such metallic nanostructures. Silver, gold and copper nanoparticles are particularly interesting due to their promising plasmonic characteristics [4-7]. They can concentrate and guide electromagnetic waves at nanoscale levels and provide extremely large localized near-field enhancement [8-10]. If compared with the other plasmonic nanoparticles, $\mathrm{Au}$ ones are characterized by a large extinction cross-section, high chemical and physical stability, high photostability, ease of surface functionalization with organic 
and biological molecules, ability to amplify electromagnetic fields and a large number of easily polarizable conduction electrons, which is a general prerequisite for preferential interaction with electromagnetic field and generation of nonlinear optical phenomena [11-16].

Metallic nanorod-like particles have recently fascinated scientists due to their distinctive optical properties which can find applications in nanoprobes, plasmonic waveguides and optical limiters [17]. In particular, Au nanorods can demonstrate a significant electric-field enhancement under plasmon resonance conditions [18]. This enhancement is located chiefly around two ends of the nanorods and can be controlled by varying the head shape. Moreover, Au nanorods can be gathered into different geometries and, as a result, the plasmonic properties of their ensemble can become stronger than those of individual nanorods [19]. That is why the localized surface plasmon resonance in the Au nanorods has been extensively studied in the recent years (see, e.g., Refs. [20, 21]).

The localized surface plasmon resonances in the metal nanostructures placed adjacent to each other are coupled together through electrostatic interaction. Plasmon coupling created in ordered nanostructures causes many interesting plasmon effects, such as huge enhancement of electric field and different collective plasmon modes [22-25]. The plasmon coupling of the Au-nanorod arrays also provides a significant increase in the electric-field enhancement if compared with the individual nanorods. These unique properties can be widely used in various fields, such as highly sensitive biological and chemical sensors, optical switches, spectroscopy, optical tweezers, and metamaterials for controlling light $[10,26,27]$. The studies of the optical properties of these ordered structures can improve our understanding of the interaction of light with matter at nanoscales. In particular, understanding of electromagnetic interactions between the plasmonic nanorods and substrates is very important for improvement of different plasmon-based devices and applications [28]. It should be noted that the dimensions of nanoparticle arrays play an important role in their optical properties due to dipolar effects and interaction with surrounding media [29]. Plasmonic light manipulation in such structures is a powerful tool for concentrating and controlling light at nanometer scales. Any advance in the mathematical models and simulations developed for controlling the properties of plasmons is crucial for selecting appropriate materials, designing devices and developing efficient manufacturing technologies in future [30].

In our previous works we have studied in detail the effect of shape on the optical properties of individual silver nanoparticles [31] and silver-based nanoshells [32]. Moreover, we have compared the spectral characteristics of individual silver-based and gold-based nanoshells [33]. Evaluation of how the laser-assisted wet chemical treatment of Ag nanoparticles affects their spatial parameter-shaping localized surface plasmon resonance has also been presented (see Ref. [34]). Furthermore, a resonance of surface-localized plasmons in a system of periodically arranged gold or silver nanowires on a dielectric substrate has been studied [35]. It has been shown that the spectral characteristics formed by the interaction of optical wave with the nanogratings based on gold and silver differ from each other. It is also worthwhile that there is a difference between the localized surface plasmon resonance and the ordinary surface plasmon resonance. In particular, metal nanoparticles are characterized by the localized surface plasmon resonance [8], while the surface plasmon resonance occurs on metal-dielectric interfaces, e.g. in plasmonic prism-based or multilayer structures [36].

In this work we focus on the linear optical properties of arrays of gold nanorods, taking into account different physical models and different variables of interaction of matter with light. To accomplish this aim, three mathematical models have been employed, which take into account the shape of particle, the ratio of irradiating light wavelength to particle size, the complex dielectric 
function of material and the dielectric constant of surrounding medium. The spectral characteristics of isolated nanorods embedded in a medium and illuminated by a plane electromagnetic wave have been modelled using a known electrostatic approximation. An effective-medium theory has been employed for calculating the refractive index of a nanocomposite composed of Au nanorods. The optical response of a two-layer structure where the first layer is a nanocomposite consisting of Au-nanorod arrays and the second one is a thin indium tin oxide (ITO) film on a glass substrate have been studied using a standard matrix method. The combination of such theories provides a simple and very efficient algorithm for calculations and predictions of optical characteristics of the plasmonic structures, including the nanoparticles deposited on a substrate and covered by additional layer. This algorithm neither involves any intricate problems nor requires many resources, which is important when designing different plasmonic devices in practice.

\section{Experimental and simulation methods}

\subsection{Experimental}

Randomly arranged aurum-nanorod arrays of a quasi-hexagonal shape were obtained using an electrochemical deposition method. Deposition of nanoparticles, especially Au, in a regime of pulse electrolysis enables a controllable synthesis of nanoparticles of a preset shape [37]. A combination of short pulses $\left(\tau_{\mathrm{on}}\right)$ with high cathode currents $\left(i_{\text {cathode }}\right)$ provides a nucleation process, with further formation of nanoparticles, whereas long pauses $\left(\tau_{\text {off }}\right)$ ensure sufficient diffusion of ions to the electrode surface. This ensures perfect conditions for the formation and growth of Au nanoparticles of uniform size and shape, which is not a case with a magnetron-sputter technique [38].

The experiment was carried out in a glass three-electrode cell $(50 \mathrm{ml})$ filled with $0.004 \mathrm{M}$ of $\mathrm{HAuCl}_{4}$ and $0.05 \mathrm{M}$ of $\mathrm{Bu}_{4} \mathrm{NClO}_{4}$ solution in $\mathrm{N}$, N-dimethylformamide at the temperature $35^{\circ} \mathrm{C}$. Au was deposited on the surface of a glass substrate covered by a thin ITO layer. The ITO-glass (Aldrich) had the resistance $70-100 \Omega / \mathrm{sq}$ and the active surface $10 \times 10 \mathrm{~mm}^{2}$. All the potentials were referred to $\mathrm{Ag} / \mathrm{AgCl}$ in a saturated $\mathrm{KCl}$ solution as to silver-chloride reference electrode. $\mathrm{A}$ gold plate was used as an anode. An electrochemical deposition process was carried out, using a pulse potentiostatic method at the voltage of $U=1.5 \mathrm{~V}$ with respect to silver chloride. The pulse time was equal to $\tau_{\text {on }}=6 \mathrm{~ms}$ and the pause time to $\tau_{\text {off }}=300 \mathrm{~ms}$. The number of pulse-pause cycles amounted to 253 . Note that a detailed methodology of the deposition of gold nanoparticles on ITO-glasses was earlier described in the works $[39,40]$.

The aspect ratio of the Au nanorods in the arrays was controlled by varying the duration of electrochemical deposition in the range from 1.17 to 1.33. A typical SEM image of the top-plane of our sample is given in Fig. 1a. The gold-nanorod particles are of different radii, which range from 40 to $80 \mathrm{~nm}$. They form randomly arranged Au-nanorod arrays with the inter-rod distances being less than the wavelength of visible light.

The absorption spectrum of the Au-nanorod array was taken at the room temperature, using a UV-VIS-NIR spectrometer from Ocean Optics USB2000 (see Fig. 1b). There was a single clear peak located at $524 \mathrm{~nm}$ and a wide additional peak at about $720 \mathrm{~nm}$. It is known that the spherical gold nanoparticles are characterized by the plasmon absorption peak near $524 \mathrm{~nm}$ [8]. Therefore, it can be argued that our structure is characterized by the plasmon absorption. However, it is well known that there are two plasmonic peaks attributed to the transverse and longitudinal resonance modes of Au nanorods [20]. Therefore the presence of a wide additional peak at about $720 \mathrm{~nm}$ indicates that the structure consists of nanorods rather than spherical nanoparticles.

Ukr. J. Phys. Opt. 2021, Volume 22, Issue 2 
The longitudinal localized surface plasmon resonance mode is associated with the electron oscillations along the length direction and the transverse localized surface plasmon resonance mode arises from the transverse electron oscillations [41]. Since our structure contains the nanorods with the heights of about $100 \mathrm{~nm}$ and different radii (see Fig. 1a), it should also be characterized by two peaks. Hence, in order to evaluate the factors affecting the absorption spectrum, a number of simulations were conducted, which are described below.
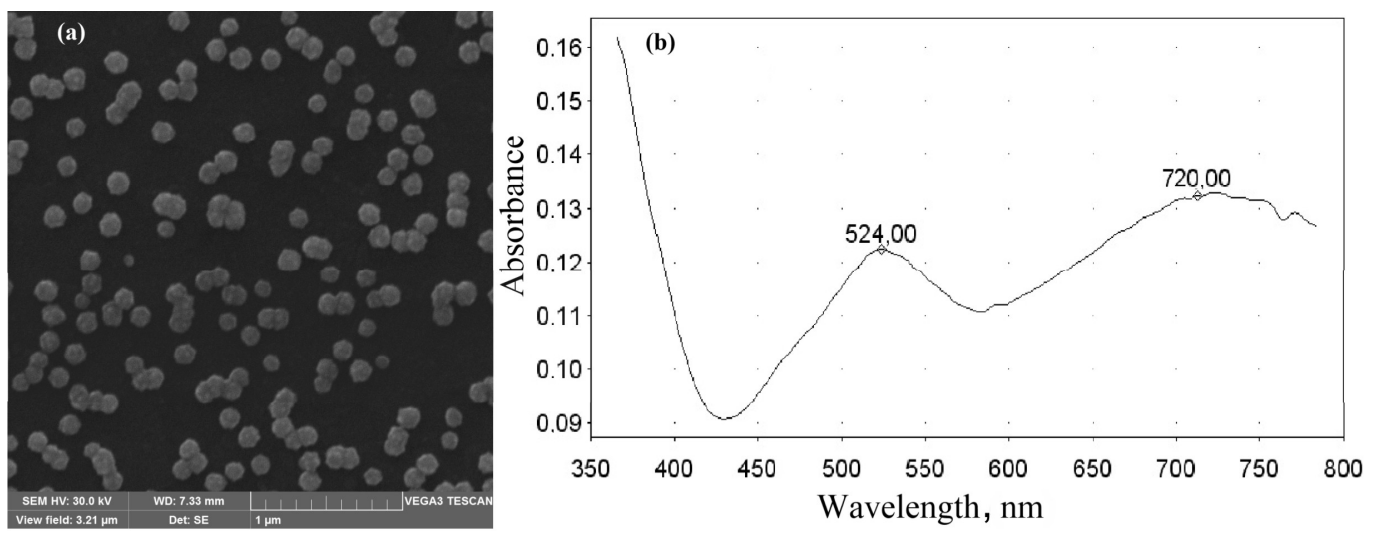

Fig. 1. SEM image of a top-plane of randomly arranged Au-nanorod arrays (a), and their absorption spectrum (b).

\subsection{Simulations}

It is known that the optical characteristics under test depend on the chemical composition of particles, their shape, size and orientation, the surrounding medium, the number of particles, as well as the polarization state and the wavelength of incident light [42]. Let us consider the spectral characteristics of the isolated nanorods embedded in some medium and illuminated by the plane wave. The plasmonic properties of the metal nanoparticles, which are small with respect to the incident-light wavelength, can be described using the electrostatic approximation. In this approximation, the electromagnetic field is considered to be the same throughout the entire particle. An elementary classical description of the plasmon resonance in a nanoparticle is based on the fact that the electromagnetic field of the incident light shifts the cloud of free electrons and creates uncompensated charges near the surface of this nanoparticle. It is enough to apply a usual dipole approximation and a Drude theory to describe at elementary level the optics of plasmon nanoparticles [43].

When it comes to the gold nanorods, the assumptions have to be extended, and the calculations become more complex. Using a Mie-Gans theory, one arrives at the conclusion that the plasmon resonance absorption splits into two bands in case of the gold nanorods. This effect arises from different surface curvatures and, hence, different restoring forces, which has been described in detail in a number of textbooks and reviews (see, e.g., Ref. [44]). As a result, the absorption $\left(C_{a b s}\right)$ and scattering $\left(C_{\text {scat }}\right)$ cross-sections are given by the following equations [20, 36]:

$$
\begin{aligned}
& C_{a b s}=k \operatorname{Im}(\alpha), \\
& C_{\text {scat }}=\frac{k^{4}}{6 \pi}|\alpha|^{2},
\end{aligned}
$$

where $\alpha$ is the polarizability and $k$ the wave vector.

In order to use this theory in calculating the plasmonic characteristics of nanorods, one has to approximate the nanorod as a prolate spheroid. This approximation does not influence the main 
conclusions regarding the plasmonic properties of the nanorods, though preserves the necessary underlying physics. Thus, the polarizability $\alpha$ (being a tensor entity) of an ellipsoid in the field parallel to its different axes can be written as follows:

$$
\begin{aligned}
& \alpha_{a}=4 \pi a b c \frac{\varepsilon_{i}-\varepsilon_{m}}{3 \varepsilon_{m}+3 L_{a}\left(\varepsilon_{i}-\varepsilon_{m}\right)}, \\
& \alpha_{b}=4 \pi a b c \frac{\varepsilon_{i}-\varepsilon_{m}}{3 \varepsilon_{m}+3 L_{b}\left(\varepsilon_{i}-\varepsilon_{m}\right)}, \\
& \alpha_{c}=4 \pi a b c \frac{\varepsilon_{i}-\varepsilon_{m}}{3 \varepsilon_{m}+3 L_{c}\left(\varepsilon_{i}-\varepsilon_{m}\right)} .
\end{aligned}
$$

Here $a, b$ and $c$ are ellipsoid semiaxes, and $\varepsilon_{i}$ and $\varepsilon_{m}$ are the dielectric functions respectively of metal and surrounding medium. Notice that the long axis of the nanorod is oriented along the axis $b$.

The geometrical factors $L$ are given by the relations

$$
\begin{aligned}
& L_{a}=\frac{a b c}{2} \int_{0}^{\infty} \frac{d q}{\left(a^{2}+q\right) f(q)}, \\
& L_{b}=\frac{a b c}{2} \int_{0}^{\infty} \frac{d q}{\left(b^{2}+q\right) f(q)}, \\
& L_{c}=\frac{a b c}{2} \int_{0}^{\infty} \frac{d q}{\left(c^{2}+q\right) f(q)},
\end{aligned}
$$

where $f(q)=\sqrt{\left(q+a^{2}\right)\left(q+b^{2}\right)\left(q+c^{2}\right)}$. Only two of the three geometrical factors $L_{a}, L_{b}$ and $L_{c}$ are independent because of the relationship $L_{a}+L_{b}+L_{c}=1$. In addition, they satisfy the inequalities $L_{a}<L_{b}<L_{c}$. Eqs. (1)-(8) testify that the absorption and scattering cross-sections change when the size and the shape of the nanorod do. Moreover, the longitudinal and transverse plasmon modes also change when the aspect ratio of the nanorod is changed.

As a first step of our modelling of the absorption cross-section, we suppose that the $\mathrm{Au}$ nanorods have the constant height $(100 \mathrm{~nm})$ and different radii, and that they are embedded in vacuum (see Fig. 2a). To be specific, we assume that the radii of the nanorods vary from 40 to $85 \mathrm{~nm}$. The refractive index of Au has been taken from Ref. [35]. It can be seen from Fig. 2a that there is a single plasmonic absorption peak. It can be explained by the fact that the axis ratio (i.e., the eccentricity) $e=a / b$ is sufficiently low. We have $e=2.5$ for the structure at the radius $40 \mathrm{~nm}$, $e=1.8$ at $55 \mathrm{~nm}, e=1.54$ at $65 \mathrm{~nm}, e=1.33$ at $75 \mathrm{~nm}$, and $e=1.17$ at $85 \mathrm{~nm}$. It is known that the absorption spectrum of a spherical nanoparticle (with the $e$ parameter being equal to unity) is characterized by a single plasmonic peak [40]. As a result, the additional plasmonic peak cannot be clearly expressed when the eccentricity is close to unity. It is confirmed by the shape of spectrum in Fig. 2 where the shift of the plasmonic absorption peak is small whenever the eccentricity is less than 2.0. To check whether our results are correct, we have performed the calculations for the nanorods having the eccentricities $e=3.75$ and $e=5.0$, which correspond respectively to the size ratios $150 \mathrm{~nm} / 40 \mathrm{~nm}$ and $200 \mathrm{~nm} / 40 \mathrm{~nm}$.

When $e$ is sufficiently large (see Fig. 2b), the absorption peak shifts towards the long-wave region. It is therefore possible to select the height and the diameter of the nanorods which provide the maximal absorption at a given wavelength.

Ukr. J. Phys. Opt. 2021, Volume 22, Issue 2 

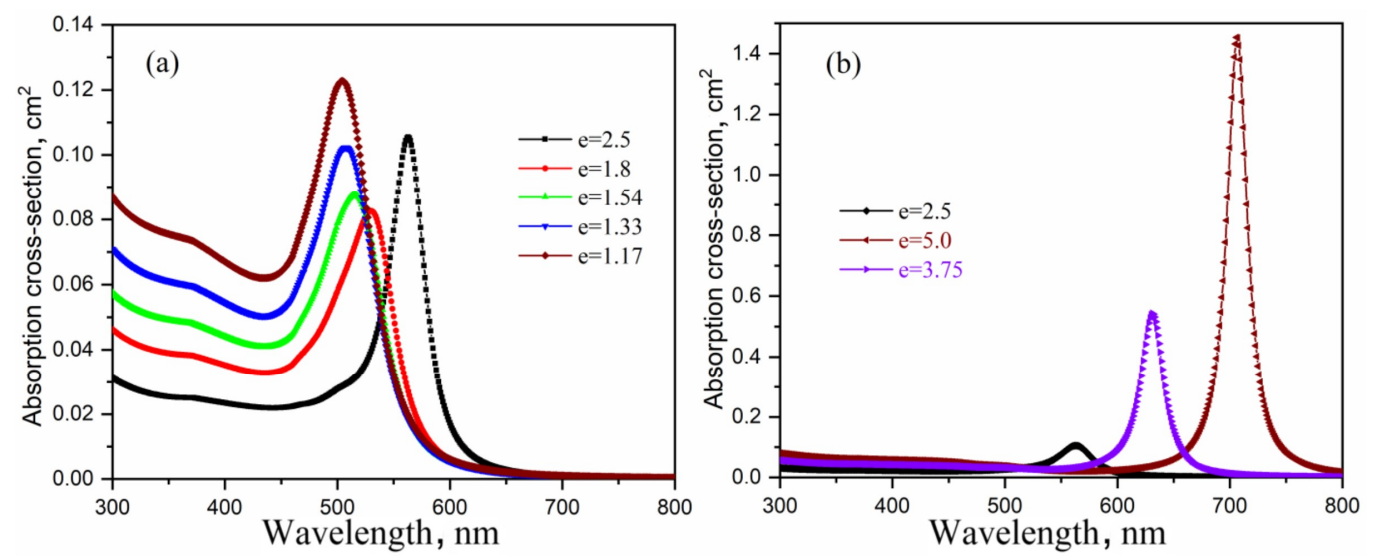

Fig. 2. Dependences of absorption cross-section for the $A u$ nanorods embedded in vacuum on the light wavelength, as calculated at different eccentricities shown in the legend: (a) nanorods of constant height $(100 \mathrm{~nm}$ ) with radius being changed from 40 to $85 \mathrm{~nm}$; (b) nanorods of constant radius $(40 \mathrm{~nm})$ with height being changed from 100 to $200 \mathrm{~nm}$.

Hence, the nanorods should in general be characterized by two absorption peaks according to the electrostatic approximation, although we observe a single peak (see Fig. 2). One can assume that the first peak, which is not sensitive to the eccentricity value, is very weak for gold in the vacuum. To confirm this assumption, we have performed similar calculations for the gold nanorods with the same size as in the previous case, which are embedded, e.g., in ITO. Note that we have used the refractive index of ITO reported in Refs. [45, 46]. Namely, the refractive index and the extinction coefficient taken from the appropriate dispersion curves have been extrapolated with seventh-order polynomials, and we have used these polynomials in our calculations. As for the main results, the absorption spectra of the Au nanorods are now characterized by two clearly distinguished absorption peaks, as seen from Fig. 3.
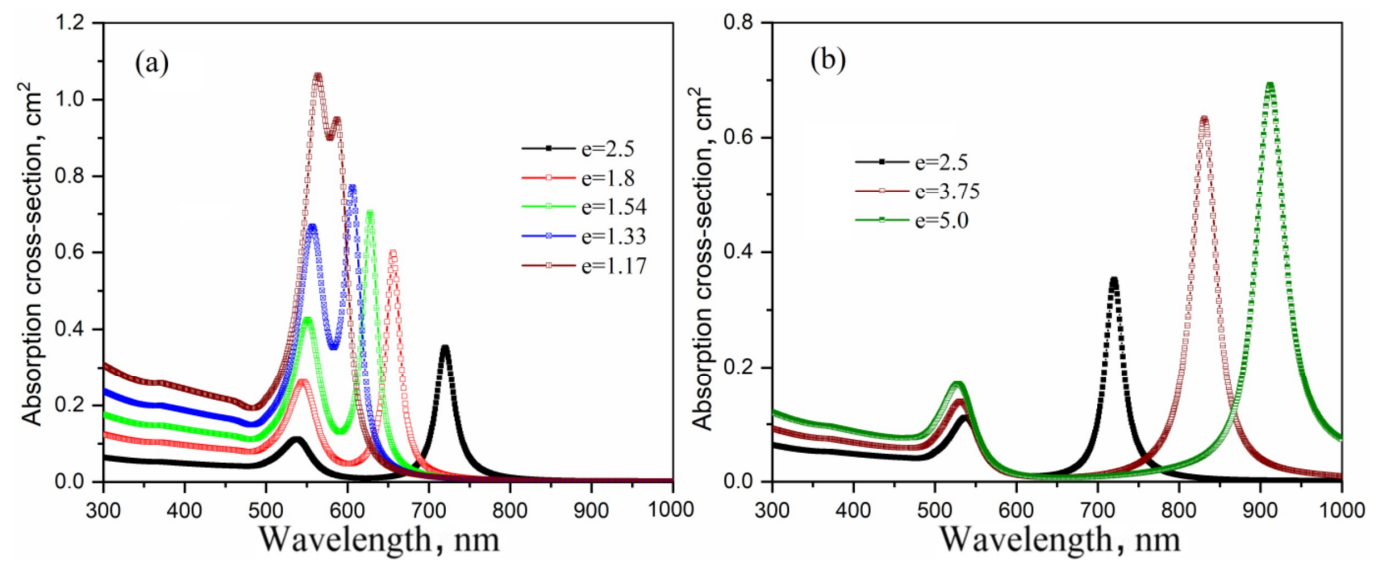

Fig. 3. Dependences of absorption cross-section of the Au nanorods embedded in ITO on the light wavelength, as calculated at different eccentricities shown in the legend: (a) nanorods of constant height $100 \mathrm{~nm}$ with radius being changed from 40 to $85 \mathrm{~nm}$; (b) nanorods of constant radius $40 \mathrm{~nm}$ with height being changed from 100 to $200 \mathrm{~nm}$.

The distance between the peaks increases with increasing axis ratio. Moreover, the results obtained by us confirm that the first peak suffers almost no shift when the size of the nanorods changes, although the second peak is very sensitive to this change. Hence, the Au nanorods are characterized by two clearly discernible absorption plasmonic peaks whenever they are embedded 
in the medium with the refractive index higher than 1.0. In addition, the absorption peak is shifted towards the near-infrared spectral region if the nanorods become higher and smaller in diameter.

\section{Results and discussion}

Our structure contains mainly the nanorods with the radii changing from 70 to $80 \mathrm{~nm}$ and the eccentricities $e=1.17 \div 1.33$. As a consequence, this structure can demonstrate only one absorption peak, which has already been confirmed by Fig. 1b. However, our subject is also the isolated nanorods embedded in the medium and illuminated by the plane light wave. Actually, we deal with the array of nanorods on the substrate covered by the ITO layer, as shown in Fig. 1a.

The structure of our sample can be schematically divided into three layers (Fig. 4). The first layer is a nanocomposite consisting of the Au nanorods, the second one is a thin ITO film with the thickness $100 \mathrm{~nm}$, and the third layer is a glass substrate. Since the eccentricities of the nanorods are about 1.0, one can suppose that their optical response can be described by the theoretical model adopted for the spherical nanoparticles. Besides, we assume that the top layer is a nanocomposite material, where air represents a host matrix and the spherical Au nanoparticles are nanoinclusions. As testified by the electron-microscopy image (Fig. 1a), the Au nanoparticles are located at the distances less than the light wavelength. Therefore, such the structure can indeed be considered as the nanocomposite material [47].

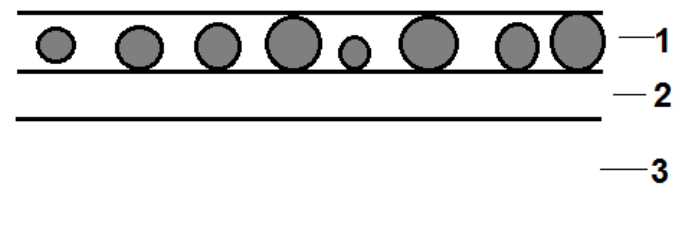

Fig. 4. Schematic representation of our sample: 1 is a nanocomposite layer consisting of Au nanoparticles, 2 an ITO layer and 3 a glass substrate.

The optical response of the nanocomposite material can be described in terms of its effective dielectric permittivity. The Maxwell-Garnett theory has earlier been successfully used to obtain a good approximation for the dependence of optical properties on the volume filling factor in case of the materials with two or more components (or phases) [48]. There are a lot of different approximations within this theory worked out in the literature [49]. Here we employ the extended Maxwell-Garnett theory so as to include the impact of nanoparticle size on the resulting response [49]. In the frame of this version of the theory, the effective permittivity for the two-constituent material includes the size effect and can be written as follows:

$$
\varepsilon_{e f f}=\varepsilon_{m} \frac{\varepsilon_{i}(1+2 f)+2 \varepsilon_{m}(1-f)+\left(\varepsilon_{m}-\varepsilon_{i}\right)(1-f) \Delta}{\varepsilon_{i}(1-f)+\varepsilon_{m}(2+f)+\left(\varepsilon_{m}-\varepsilon_{i}\right)(1-f) \Delta},
$$

where $\varepsilon_{m}$ and $\varepsilon_{i}$ are the dielectric permittivities respectively of the host matrix and the inclusions, and $f$ denotes the volume filling factor. The $\Delta$ parameter describing the size effect in the effective dielectric permittivity is expressed as

$$
\Delta=\left(\frac{\sqrt{\varepsilon_{i}} 2 \pi R}{\lambda}\right)^{2}+\frac{2}{3} i\left(\frac{\sqrt{\varepsilon_{i}} 2 \pi R}{\lambda}\right)^{3},
$$

where $R$ is the radius of nanoparticle.

Ukr. J. Phys. Opt. 2021, Volume 22, Issue 2 
Now that the dielectric permittivities of all the layers are given, it is possible to calculate the absorption coefficient of such a three-layer structure, using the matrix method. This method is widely used for calculating the optical properties of multilayer structures [35]. Here each layer is described by a characteristic matrix:

$$
M_{j}=\left(\begin{array}{cc}
\cos \delta_{j} & \left(\frac{i}{\eta_{j}}\right) \sin \delta_{j} \\
i \eta_{j} \sin \delta_{j} & \cos \delta_{j}
\end{array}\right),
$$

where $\delta_{j}=2 \pi / \lambda\left(\eta_{j} d_{j} \cos \theta_{j}\right), \eta_{j} d_{j} \cos \theta_{j}$ implies the effective optical thickness of the layer $j$ for the refraction angle $\theta_{j}, d_{j}$ the geometrical thickness of layer, and $\eta_{j}$ its effective refractive index.

The amplitudes of the reflection $(r)$ and transmission $(t)$ coefficients of the multilayer structure are expressed using the electric $\left(E_{0}\right)$ and magnetic $\left(H_{0}\right)$ vectors in the incident medium:

$$
r=\frac{\eta_{0} E_{0}-H_{0}}{\eta_{0} E_{0}+H_{0}}, \quad t=\frac{2 \eta_{0}}{\eta_{0} E_{0}+H_{0}},
$$

with $\left(\begin{array}{c}E_{0} \\ H_{0}\end{array}\right)=M\left(\begin{array}{c}1 \\ \eta_{S}\end{array}\right)$. As a result, the absorption of the multilayer structure is given by

$$
A=1-\frac{\eta_{S}}{\eta_{0}}|t|^{2}-|r|^{2}
$$

where $\eta_{s}$ and $\eta_{0}$ are the effective refractive indices of the substrate and the surrounding medium, respectively.

Fig. 5 shows the calculated absorption spectra for our three-layer structure at different filling factors. The glass with the refractive index 1.5152 is used as a substrate in our calculations. The gold nanoparticles have the radius $65 \mathrm{~nm}$, while the thickness of the nanocomposite layer is selected to be $130 \mathrm{~nm}$. Finally, the angle of incidence is equal to $0^{\circ}$.

As seen from Fig. 5, the absorption amplitude in the spectrum increases with increasing nanoparticle-filling factor. The width of the absorption peak then increases, too. However, the position of the plasmon absorption peak remains stable and practically does not shift. Thus, we are

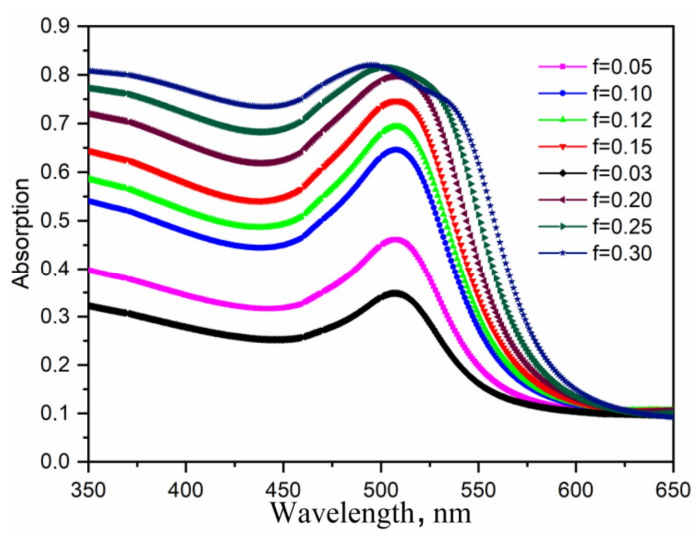

Fig. 5. Absorption spectra of our two-layer structure, in which the first layer is a nanocomposite consisting of Au nanorods and the second one is a thin ITO film on glass substrate. Different curves correspond to different Aunanorod filling factors (see the legend). 
in a position to estimate the filling factor of nanoinclusions in the composite layer, using the experimental results presented in Fig. $1 \mathrm{~b}$ and the data calculated theoretically (see Fig. 5). All the further calculations have been performed for the filling factor 0.20 , which corresponds to the sample presented in Fig. 1.

It is well known that the plasmon resonance in the Au nanorods is highly sensitive to the surrounding medium. Then, in order to study the impact of properties of the host matrix of the composite layer on the resulting response of the whole structure, we calculate the absorption spectra of the multilayer structure with different refractive indices of the host matrix (Fig. 6a). Since the Au nanoparticles interact with the ITO layer and any real model of air must include some impurities, the refractive index can also be affected by these factors.
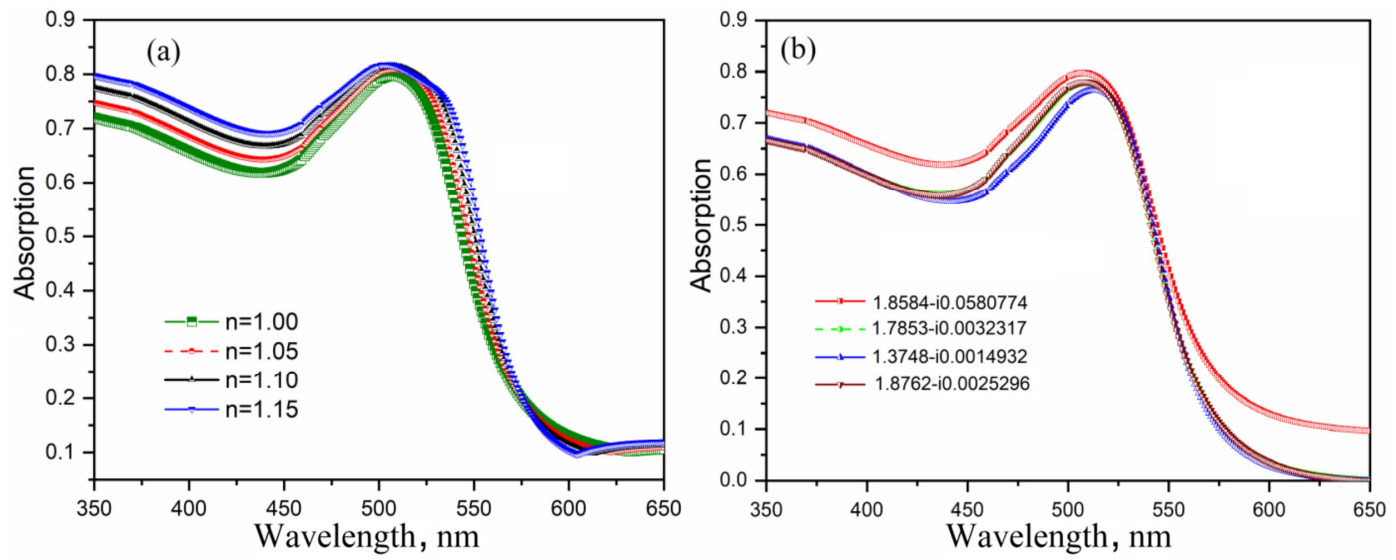

Fig. 6. Absorption spectra calculated for our two-layer structure, in which the first layer is a nanocomposite consisting of Au nanorods and the second one is a thin ITO film on glass substrate. Different curves in panels (a) and (b) correspond respectively to different refractive indices of the matrix of nanocomposite layer and to different refractive indices of the ITO layer.

The refractive index of the host matrix of the nanocomposite layer has been varied from 1.00 to 1.15 , with the steps of 0.05 . The amplitude and the width of the absorption peak increase with increasing refractive index of the matrix. Besides, there is a slight shift of the plasmon absorption peak towards the infrared region.

It is worth noting that the absorption spectrum undergoes transformations when the refractive index of the matrix of the composite layer approaches the value of 1.15. As a result, the refractive index of the nanocomposite matrix affects notably the resulting characteristics of the overall structure. In addition to the nanocomposite layer, the parameters of the ITO layer can also affect the optical response of the structure. Therefore, the absorption spectra of our structure have been calculated at different refractive indexes of ITO (see Fig. 6b). Four values of the complex refractive index have been selected in our calculations: $1.8584-i 0.0580774,1.7853-i 0.0032317$, $1.3748-i 0.0014932$ (see Refs. $[45,46]$ ), and 1.8762-i0.0025296 (se Ref. [50]). Fig. 6b demonstrates that the refractive index of ITO influences both the amplitude and the width of the absorption peak. Note that the influence of the ITO refractive index on the absorption-peak position is especially clear at the first and third values of the refractive index.

Another critical factor affecting the parameters of nanocomposite materials is the size of nanoparticles. Therefore, as a next step, we have studied the effect of nanoparticle radius on the resulting optical response. Fig. 7a presents the simulation data for our structure at different nanoparticle radii $R$ (and, accordingly, different thicknesses of the composite layer, since the latter 
is equal to the diameter of nanoparticles). The nanoparticle radii in the range from 45 to $75 \mathrm{~nm}$ (with the increments of $5 \mathrm{~nm}$ ) have been taken in our calculations, whereas the thickness of the composite layer has been varied from 90 to $150 \mathrm{~nm}$. As seen from Fig. 7, the amplitude and the width of the absorption peak increases with increasing radius of nanoparticles and thickness of nanocomposite layer. At the same time, these parameters hardly affect the spectral position of the absorption peak.
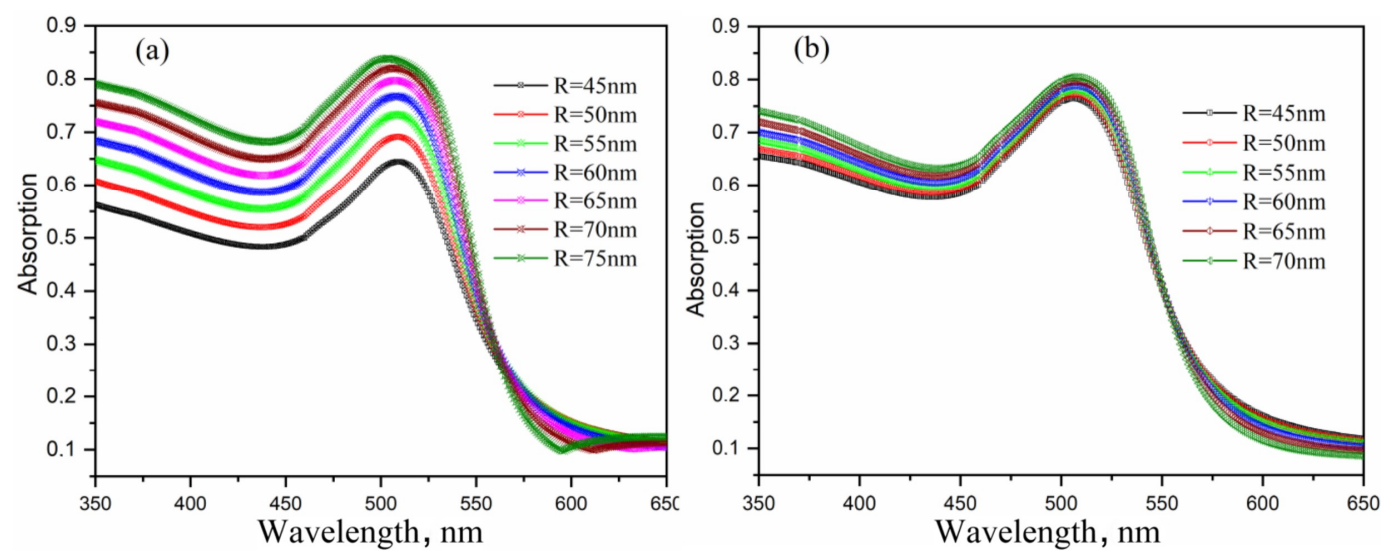

Fig. 7. Absorption spectra calculated for our two-layer structure, in which the first layer is a nanocomposite consisting of Au nanorods and the second one is a thin ITO film on glass substrate. Different curves in panels (a) and (b) correspond respectively to different nanoparticle radii (and thicknesses of composite layer) and to different nanoparticle radii at a constant thickness of composite layer $(130 \mathrm{~nm})$.

As seen from the electron microscopy data (Fig. 1a), the radii of the nanorods in the layer are different and, therefore, one can assume that these nanorods have different heights. Then one can suppose that the nanocomposite layer has a certain (fixed) thickness, but the inclusions are of different sizes. The dependences of the absorption spectra on the size of nanoinclusions at the constant layer thickness $(130 \mathrm{~nm}$ ) have been calculated (see Fig. 7b). It is evident that the size of the nanoinclusions affects only the amplitude of the absorption peak, although its impact is not significant.

\section{Conclusions}

Let us summarize the main results obtained in the present work. At first, we conclude that the $\mathrm{Au}$ nanoparticles of preset shapes can be synthesised successfully with the pulse electrolysis method. Then, our data confirms that the longitudinal plasmon resonance overwhelms the other resonances in the case of Au nanorods deposited on the substrates with small dielectric constants (e.g., glasses). As a result, the absorption spectra are characterized by a single plasmonic peak. It is important that our results indicate for the first time the following fact: one can apply the Maxwell-Garnett theory and the matrix method for calculating and predicting the optical characteristics of the structures that consist of nanoparticles deposited on a substrate covered by an additional layer, using some assumptions about the composition and the thickness of nanocomposite layer. It has also been shown that the concentration of nanoparticles on the surface can be estimated using the simulation data.

It should be noted that the experimental spectra are slightly different from the calculated ones. This may originate from the fact that, while performing our calculations, we have taken tabular parameters of the materials under test, although these parameters are somewhat different in any practical situation. The structures studied by us can be successfully applied in various practical 
fields, because one can achieve the maximal absorption at a given wavelength by simply changing the geometry of nanorods.

Nowadays, only time-consuming and high-cost top-down methods can produce the nanorod arrays with controlled nanorod geometries and orientations. Therefore, facile and easily controllable methods for assembling the colloidal nanorods are strongly desired for future applications. The pulse electrolysis is one of those methods.

\section{Acknowledgements}

This study was funded by the Ministry of Education and Science of Ukraine under the Projects "Controlled electrochemical synthesis of metal nanoparticles and nanostructured materials" (\#0118U000268) and "Nanostructured interfaces based on non-toxic materials for practical applications" (\#0120U100675)

\section{References}

1. Kasani S, Curtin K and Wu N, 2019. A review of 2D and 3D plasmonic nanostructure array patterns: fabrication, light management and sensing applications. Nanophotonics. 8: 2065-2089.

2. Ma X, Song S, Kim S, Kwon M S, Lee H, Park W and Sim S J, 2019. Single gold-bridged nanoprobes for identification of single point DNA mutations. Nature Commun. 10: 1-13.

3. Striebel M, Wrachtrup J and Gerhardt I, 2017. Absorption and extinction cross sections and photon streamlines in the optical near-field. Sci. Rep. 7: 1-13.

4. Zaman Q, Souza J, Pandoli O, Costa K Q, Dmitriev V, Fulvio D and Del Rosso T, 2019. Twocolor surface plasmon resonance nanosizer for gold nanoparticles. Opt. Express. 27: 3200-3216.

5. Mogensen K B and Kneipp K, 2014. Size-dependent shifts of plasmon resonance in silver nanoparticle films using controlled dissolution: monitoring the onset of surface screening effects. J. Phys. Chem. C. 118: 28075-28083.

6. Lesyuk R, Klein E, Yaremchuk I and Klinke C, 2018. Copper sulfide nanosheets with shapetunable plasmonic properties in the NIR region. Nanoscale. 10: 20640-20651.

7. Rao K S, Ganeev R A, Zhang K, Fu Y, Boltaev G S, Maurya S K and Guo C, 2019. Comparative analyses of optical limiting effects in metal nanoparticles and perovskite nanocrystals. Opt. Mater. 92: 366-372.

8. Maier S A. Plasmonics: fundamentals and applications (Springer Science \& Business Media, 2007).

9. Gramotnev D K and Bozhevolnyi S I, 2014. Nanofocusing of electromagnetic radiation. Nature Photonics. 8: 13.

10. Huang Y, Zhang X, Ringe E, Hou M, Ma L and Zhang Z, 2016. Tunable lattice coupling of multipole plasmon modes and near-field enhancement in closely spaced gold nanorod arrays. Sci. Rep. 6: 23159.

11. Zhang Y, Sun H, Zhang S, Li S, Wang X, Zhang X and Guo Z, 2019. Enhancing luminescence in all-inorganic perovskite surface plasmon light-emitting diode by incorporating Au-Ag alloy nanoparticle. Opt. Mater. 89: 563-567.

12. Gao Q, Zhang X, Duan L, Li X and Lü W, 2019. Au nanoparticle-decorated $\mathrm{TiO}_{2}$ nanorod array for plasmon-enhanced quantum dot sensitized solar cells. Superlatt. Microstruct. 129: $185-192$. 
13. Yang J, Liu Z, Hu Z, Zeng F, Zhang Z, Yao Y and Pi M, 2019. Enhanced single-mode lasers of all-inorganic perovskite nanocube by localized surface plasmonic effect from $\mathrm{Au}$ nanoparticles. J. Lumin. 208: 402-407.

14. Amendola V, Pilot R, Frasconi M, Marago O M and Iati M A, 2017. Surface plasmon resonance in gold nanoparticles: a review. J. Phys.: Condens. Matter. 29: 203002.

15. Lim D K, Barhoumi A, Wylie R G, Reznor G, Langer R S and Kohane D S, 2013. Enhanced photothermal effect of plasmonic nanoparticles coated with reduced graphene oxide. Nano Lett. 13: 4075-4079.

16. Alkilany A M, Lohse S E and Murphy C J, 2012. The gold standard: gold nanoparticle libraries to understand the nano-bio interface. Acc. Chem. Res. 46: 650-661.

17. Smitha S L, Gopchandran K G, Smijesh N and Philip R, 2013. Size-dependent optical properties of Au nanorods. Prog. Nat. Sci.: Mater. Int. 23: 36-43.

18. Ming T, Zhao L, Yang Z, Chen H, Sun L, Wang J and Yan C, 2009, Strong polarization dependence of plasmon-enhanced fluorescence on single gold nanorods. Nano Lett. 9: 3896-3903.

19. Lan X and Wang Q, 2016. Self-assembly of chiral plasmonic nanostructures. Adv. Mater. 28: 10499-10507.

20. Chen H, Shao L, Li Q and Wang J, 2013, Gold nanorods and their plasmonic properties. Chem. Soc. Rev. 42: 2679-2724.

21. Kravets V G, Kabashin A V, Barnes W L and Grigorenko A N, 2018. Plasmonic surface lattice resonances: a review of properties and applications. Chem. Rev. 118: 5912-5951.

22. Dong J, Zhao X, Gao W, Han Q, Qi J, Wang Y and Sun M, 2019. Nanoscale vertical arrays of gold nanorods by self-assembly: Physical mechanism and application. Nanoscale Res. Lett. 14: 118.

23. Hong S, Shuford K L and Park S, 2011, Shape transformation of gold nanoplates and their surface plasmon characterization: triangular to hexagonal nanoplates. Chem. Mater. 23: 2011-2013.

24. Liu B, Yan H, Stosch R, Wolfram B, Bröring M, Bakin A and Lemmens P, 2017. Modelling plexcitons of periodic gold nanorod arrays with molecular components. Nanotechnol. 28: 195201.

25. Payne E K, Shuford K L, Park S, Schatz G C and Mirkin C A, 2006. Multipole plasmon resonances in gold nanorod. J. Phys. Chem. B. 110: 2150-2154.

26. McMillan B G, Berlouis L E, Cruickshank F R, Pugh D and Brevet P F, 2005. Transverse and longitudinal surface plasmon resonances of a hexagonal array of gold nanorods embedded in an alumina matrix. Appl. Phys. Lett. 86: 211912.

27. Lee K C, Chen Y H, Lin H Y, Cheng C C, Chen P Y, Wu T Y and Chang C W, 2015. Plasmonic gold nanorods coverage influence on enhancement of the photoluminescence of two-dimensional $\mathrm{MoS}_{2}$ monolayer. Sci. Rep. 5: 16374.

28. Chen H, Ming T, Zhang S, Jin Z, Yang B and Wang, J, 2015. Effect of the dielectric properties of substrates on the scattering patterns of gold nanorods. ACS Nano. 5: 4865-4877.

29. Liu Y, Begin-Colin S, Pichon B P, Leuvrey C, Ihiawakrim D, Rastei M and Bigot J Y, 2014. Two dimensional dipolar coupling in monolayers of silver and gold nanoparticles on a dielectric substrate. Nanoscale. 6: 12080-12088.

30. Jia C, Li X, Xin N, Gong Y, Guan J, Meng L and Guo X, 2016. Interface-engineered plasmonics in metal/semiconductor heterostructures. Adv. En. Mater. 6: 1600431. 
31. Varyshchuk V, Bulavinets T, Yaremchuk I and Bobitski Y, 2018. The shape effect on the optical properties of metallic nanoparticles. In: $14^{\text {th }}$ International Conference on Advanced Trends in Radioelecrtronics, Telecommunications and Computer Engineering (TCSET), p. 458-461.

32. Bulavinets T, Yaremchuk I, Fitio V and Bobitski Y, 2019. Spectral characteristics of the titanium dioxide-silver nanoshells under localized surface plasmon resonance. In: IEEE $2^{\text {nd }}$ Ukrainian Conference on Electrical and Computer Engineering (UKRCON), p. 762-765.

33. Bulavinets T, Yaremchuk I., Fitio V, Barylyak A and Bobitski Y, 2019. Comparison of spectral characteristics of $\mathrm{TiO}_{2} @ \mathrm{Ag}$ and $\mathrm{Ag} @ \mathrm{TiO}_{2}$ core-shell nanoparticles. In: International Conference on Information and Telecommunication Technologies and Radio Electronics (UkrMiCo), p. 1-4.

34. Bulavinets T, Kulpa-Greszta M, Tomaszewska A, Kus-Liśkiewicz M, Bielatowicz G, Yaremchuk I, Barylyak A, Bobitski Y and Pązik R, 2020. Efficient NIR energy conversion of plasmonic silver nanostructures fabricated with the laser-assisted synthetic approach for endodontic applications. RSC Adv. 10: 38861-38872.

35. Fitio V, Yaremchuk I, Vernyhor O and Bobitski Y, 2018. Resonance of surface-localized plasmons in a system of periodically arranged gold and silver nanowires on a dielectric substrate. Appl. Nanosci. 8: 1015-1024.

36. Fitio V, Yaremchuk I and Bobitski Y, 2011. Optical excitation of surface plasmon polariton and waveguide modes resonances on prismatic structures. Opt. Applicata. 41: 929-939.

37. Saldan I, Dobrovetska O, Sus L, Makota O, Pereviznyk O, Kuntyi O and Reshetnyak O, 2018. Electrochemical synthesis and properties of gold nanomaterials. J. Solid State Electrochem. 22: $637-656$.

38. Stankevičius E, Garliauskas M, Laurinavičius L, Trusovas R, Tarasenko N and Pauliukaitė R, 2019. Engineering electrochemical sensors using nanosecond laser treatment of thin gold film on ITO glass. Electrochim. Acta. 297: 511-522.

39. Sus L, Okhremchuk Y, Saldan I, Kuntyi O, Reshetnyak O and Korniy S, 2015. Controlled gold deposition by pulse electrolysis. Mater. Lett. 139: 296-299.

40. Kuntyi O I, Sus LV, Kornii S A and Okhremchuk E V, 2016, Electrodeposition of gold nanoparticles in dimethylformamide solutions of $\mathrm{H}\left[\mathrm{AuCl}_{4}\right]$. Mater. Sci. 51: 885-889.

41. Brioude A, Jiang X C and Pileni M P, 2005. Optical properties of gold nanorods: DDA simulations supported by experiments. J. Phys. Chem. B. 109: 13138-13142.

42. Bohren C F and Huffman D R. Absorption and scattering of light by small particles (John Wiley \& Sons, 2008).

43. Dykman L and Khlebtsov N, 2012. Gold nanoparticles in biomedical applications: recent advances and perspectives. Chem. Soc. Rev. 41: 2256-2282.

44. Prescott S W and Mulvaney P, 2006. Gold nanorod extinction spectra. J. Appl. Phys. 99: 123504.

45. https://refractiveindex.info/?shelf=other\&book=In2O3-SnO2\&page=Konig-EMA

46. König T A, Ledin P A, Kerszulis J, Mahmoud M A, El-Sayed M A, Reynolds J R and Tsukruk $\mathrm{V} \mathrm{V}$, 2014. Electrically tunable plasmonic behavior of nanocube-polymer nanomaterials induced by a redox-active electrochromic polymer. ACS Nano. 8: 6182-6192.

47. Prasad P N. Nanophotonics (John Wiley \& Sons, 2004).

48. Levy O and Stroud D, 1997. Maxwell Garnett theory for mixtures of anisotropic inclusions: Application to conducting polymers. Phys. Rev. B. 56: 8035.

Ukr. J. Phys. Opt. 2021, Volume 22, Issue 2 
49. Ruppin R, 2000. Evaluation of extended Maxwell-Garnett theories. Opt. Commun. 182: 273-279.

50. Moerland R J and Hoogenboom J P, 2016. Subnanometer-accuracy optical distance ruler based on fluorescence quenching by transparent conductors. Optica. 3: 112-117.

Yaremchuk I., Pidluzhna A., Stakhira P., Kuntyi O., Sus L., Savaryn V., Kostruba A., Fitio V. and Bobitski Y. 2021 Surface-localized plasmon resonance in a system of randomly arranged gold nanorods on a dielectric substrate. Ukr.J.Phys.Opt. 22: 69-82. doi: 10.3116/16091833/22/2/69/2021

Анотація. Матрииі наностержнів золота квазігексагональної форми успішно одержано за методом електрохімічного висадження. Досліджені їхні оптичні властивості. Проаналізовано оптичний відгук двочарової структури, в якій периий шар $\epsilon$ нанокомпозитом, що складається з наностержнів Аи, а інший - тонкою плівкою оксиду олова індію на скляній підкладиі. У рамках електростатичного наближення виконано моделювання залежності перетину поглинання наностержнів Аи від довжини світлової хвилі для різних ексиентриситетів. Показано, що поздовжній плазмонний резонанс переважає над іншими типами резонансів у наностержнях Аи, нанесених на скляні підкладки. Для прогнозування оптичних характеристик всієї структури використано теорію Максвелла-Гарнета та матричний метод. Продемонстровано, що на основі відповідних результатів моделювання можна оцінити конщентрачію наностержнів на поверхні. Крім того, потрібне поглинання на заданій довжині хвилі можна одержати шляхом зміни геометрії наностержнів. Зокрема, ми спостерігали зсув піку поглинання в напрямку ближньоі інфрачервоної області, якщо наностержні набувають достатню висоту та стають меншими в діаметрі. 